\title{
Glioblastoma, bone sarcoma, and liver cancer: tough battles rage on for some tumors
}

\author{
Jane de Lartigue, $\mathrm{PhD}$
}

I mprovements in our understanding of the molecular mechanisms of cancer combined with advances in genome sequencing have provided revolutionary new therapeutic options for several hard-to-treat tumors in recent decades. For other challenging tumor types these advancements have served only to highlight their significant complexity and, despite the development of novel treatments, there has been limited improvement in prognosis.

\section{Aggressive GBM remains incurable despite FDA approvals}

Though rare, glioblastoma (GBM) are the most common malignant primary brain tumors. These highly aggressive tumors have a dismal prognosis (median survival from diagnosis is just over a year) $)^{1,2}$ and are notoriously hard to treat for many reasons, including their location, poor response to therapy, and complex and heterogeneous molecular make-up.

Unlike most other tumors, which can often be cured by surgical resection if caught early enough, it is difficult to completely remove GBM safely. Nevertheless, surgery in combination with chemotherapy and radiation therapy (RT) remains standard of care (SOC), reflecting the limited treatment options available.

GBM is among the most highly vascularized solid tumors, characterized by extensive angiogenesis and an abnormal vasculature, and anti-angiogenic therapies targeting the vascular endothelial growth factor (VEGF) and its receptor (VEGFR) have been intensely investigated. Based on the demonstration of improved response rates, bevacizumab received regulatory approval by the US Food and Drug Administration in 2009 for the treatment of recurrent GBM. ${ }^{3}$ It remains the only targeted therapy approved by the agency and no agent to date has demonstrated improved survival in this setting.

Meanwhile, the use of bevacizumab in the firstline setting has stirred up significant debate, after

contrasting reports from 2 phase 3 trials. Both of the trials evaluated the addition of bevacizumab to SOC and showed similar improvement in progression-free survival (PFS), with no significant effect on overall survival (OS). However, a marked difference was observed in the impact of bevacizumab on quality of life and performance status, with one trial showing an improvement and the other a worsening. It will be an important discrepancy to unravel because it could have an impact on the potential utility of bevacizumab in this setting. ${ }^{4,5}$

Bevacizumab continues to be evaluated in clinical trials in combination with other agents (Online, Table 1), and other anti-angiogenic therapies have also been tested in GBM. Cediranib is a small molecule inhibitor of VEGFR, which, despite promising activity in early clinical trials, failed to demonstrate a survival benefit in the recent phase 3 REGAL trial, alone or in combination with lomustine. ${ }^{6}$ Cilengitide is an integrin inhibitor; integrins mediate communication between GBM cells and the brain microenvironment and play an important role in angiogenesis as well as motility and invasiveness. This agent also reached phase 3 development but failed to improve patient outcomes when combined with temozolomide and RT in the recently reported CENTRIC trial. ${ }^{7}$

A number of other targeted therapies have been evaluated in GBM (Figure 1) and have, likewise, demonstrated only modest therapeutic activity. The most prominent example is epidermal growth factor receptor (EGFR)-targeted drugs; amplification and overexpression of EGFR is observed in more than half of GBM cases and about half of those are caused by a mutant form of the receptor, EGFRvIII, which leads to constitutive activation of kinase activity, ${ }^{8,9}$

Several novel therapeutic strategies may offer a glimmer of hope. Researchers have created a "cancer hat" that passes low intensity, intermediate frequency alternating electric fields through the brain.

JCSO 2015;13:162-166. @2014 Frontline Medical Communications. DOI 10.12788/jcso.0129. 


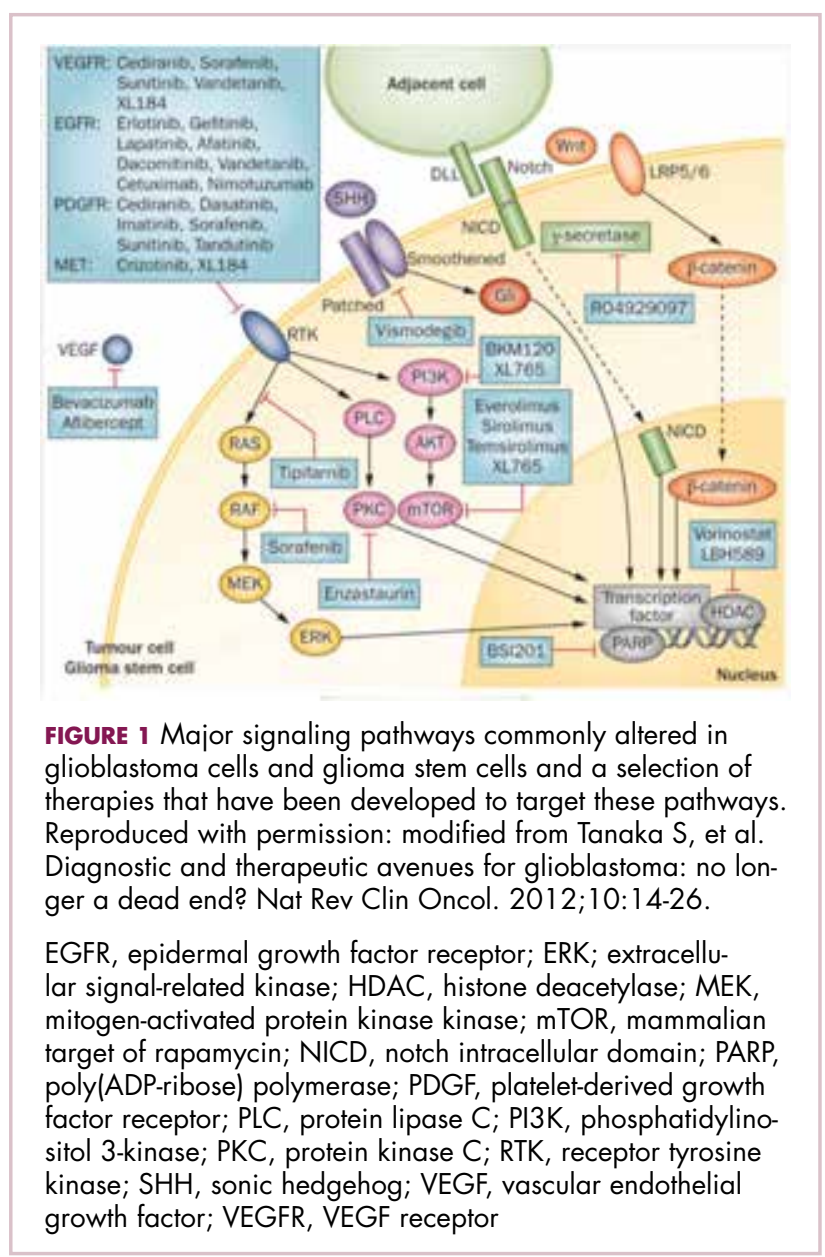

It's hypothesized that these fields block the formation of the mitotic spindle and prevent proliferation and differentiation of dividing cells. The NovoTTF-100A/Optune device was developed in patients with GBM and, after a phase 3 trial demonstrated comparable efficacy with physician's choice chemotherapy, it was approved by the FDA in $2011 .{ }^{10}$ Numerous clinical trials of NovoTTF$100 \mathrm{~A}$ are ongoing and preliminary results from a phase 3 trial in combination with temozolomide in patients with newly diagnosed GBM, which were recently reported at the annual meeting of the Society for Neuro-Oncology (SNO), showed improved PFS and OS. ${ }^{11}$

Immunotherapies, which have taken center stage for cancer treatment in recent years following increased appreciation of the intricate relationship between the immune system and cancer, have also emerged as a potentially effective treatment option for GBM. The major focus has been on vaccines (Online, Table 2), predominantly those based on dendritic cells (DCs). ${ }^{12}$ Rindopepimut targets the EGFRvIII mutant and the phase 3 ACT IV study in patients with newly diagnosed GBM completed enrollment in late 2014. Interim results from the phase $2 \mathrm{ReACT}$ study in patients with relapsed GBM were presented at the 2014 SNO meeting; combination of rindopepimut and bevacizumab improved survival outcomes in patients with both bevacizumabnaive and -refractory tumors. Based on these and other positive phase 2 data, the FDA has granted this vaccine breakthrough therapy designation. ${ }^{13}$

\section{Moving beyond the therapeutic plateau in bone sarcomas}

Fewer than $1 \%$ of all cancers are primary bone tumors (sarcomas); most common are osteosarcoma, which arises in the osteoid tissue; chondrosarcoma (CS), in the cartilaginous tissues; and Ewing sarcoma (ES), which forms in the bone and soft tissue. ${ }^{14}$

Rarity is among the many factors that makes these tumors difficult to treat, and as with GBM, their location makes surgical resection technically challenging and the survival rate with surgery alone is low. Routine use of chemotherapy has vastly improved outcomes, with a cure rate of $60 \%-70 \%$ for osteosarcoma and 50\%-60\% for ES. ${ }^{15}$ However, CS is very often refractory to chemotherapy and outcomes depend on the histologic grade and specific type of CS. Although the development of chemotherapy was a major advancement for the treatment of osteosarcoma and ES, a therapeutic plateau has been reached. A range of novel therapeutic strategies have been evaluated and, to date, none has further improved survival (Online Table 3; Figure 2). This is particularly problematic for the majority of patients who are diagnosed at more advanced stages of disease that are not surgically treatable and who respond poorly to chemotherapy.

The genomic complexity of bone sarcomas is another significant challenge to their treatment. Apart from ES, which is characterized by translocations in the EWSR1 gene, they are extremely heterogeneous and few broadly targetable driver mutations have been identified. This complexity was highlighted in a recent study. ${ }^{16}$ Most pediatric cancers have a low somatic mutation rate (around 0.1 mutations $/ \mathrm{mega}^{-}$ base $[\mathrm{Mb}])$, but in the aforementioned study osteosarcoma was found to have 1.2 mutations/Mb, which is comparable with some adult tumors. ${ }^{16}$

EWSR1/FLI-1 is the most common activating translocation of the EWSR1 gene and plays a significant role in the formation of ES. The insulin-like growth factor receptor (IGF1R) pathway is a major downstream target of activated EWSR1 and is also upregulated in osteosarcoma, thus representing a promising therapeutic target for both cancer types. ${ }^{17-19}$

Clinical trials of IGF1R inhibitors showed notable responses among a small number of patients with advanced, heavily pretreated disease, but these were short-lived and insufficient to advocate monotherapy. One possible expla- 


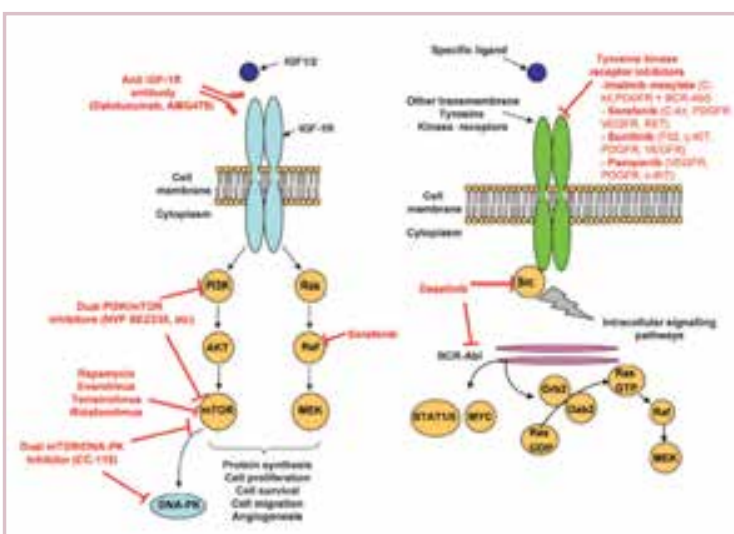

FIGURE 2 Signaling pathways targeted by experimental sarcoma therapies. There are currently no FDA-approved targeted therapies for the treatment of bone sarcomas. The IGFIR shown on the left is one of the most promising drug targets, particularly in Ewing sarcoma. A number of monoclonal antibodies targeting this receptor have been evaluated. Responses, though dramatic, are typically short-lived. A number of proteins activated downstream of IGFIR are also being targeted and there is hope for combination therapy. Multi-targeted tyrosine kinase inhibitors have also been heavily investigated, several examples of which are shown on the right. Reproduced with permission from Heymann D, et al. ${ }^{18}$

DNA-PK, DNA-dependent protein kinase; FDA, US Food and Drug Administration; Flt3, fms-related tyrosine kinase 3; Gab2, Grb2-associated binding protein 2; GDP, guanosine diphosphate; Grb2, growth factor receptor-bound protein 2; GTP, guanosine triphosphate; IGF 1/2, insulin-like growth factor $1 / 2$; IGF 1R, insulin-like growth factor receptor 1 ; MEK, mitogen-activated protein kinase kinase; mTOR, mammalian target of rapamycin; PDGFR, platelet-derived growth factor receptor; PI3K, phosphatidylinositol 3-kinase; STAT1/5, signal transducer and activator of transcription 1/5; VEGFR, vascular endothelial growth factor receptor

nation for the limited response is that downstream pathways that compensate for the loss of IGF1R activity are activated. Activation of the mammalian target of rapamycin (mTOR) was identified as a potential mechanism of resistance and mTOR inhibitors have also been extensively investigated, both as monotherapy and in combination with IGF1R inhibitors. ${ }^{17}$

Some studies have found that when mTOR inhibitors were added to IGF1R therapy, response rates improved and the duration of response was significantly extended, although other studies reported no objective responses. mTOR monotherapy has also proven disappointing despite positive results from early clinical trials.

Although these agents are potentially promising in select populations, without the ability to define the patient populations, development of these agents has stalled and the future of this therapeutic strategy remains unclear. A limited number of clinical trials are ongoing, and researchers are focusing on identifying biomarkers to aid in patient selection.
These studies have also highlighted the importance of understanding patients who seem to have exceptional responses to targeted therapies. Until recently, reports of these "miraculous" outcomes for a select few patients have been largely ignored, and drugs that fail to show improvement for a large number of patients are considered failures. Technological advances have made it possible to unravel the underlying molecular mechanisms of this response. ${ }^{20}$

Studying exceptional responders could prove particularly useful in identifying novel therapies for patients with rare, hard-to-treat tumors such as bone sarcomas. Sequencing studies of exceptional responders from trials of IGF1R and mTOR inhibitor therapy are ongoing and could prove critical to understanding whether further development of these agents should be pursued. ${ }^{21,22}$

\section{Hepatocellular carcinoma: failure to improve on sorafenib success}

Unlike GBM and bone sarcomas, liver cancer is quite common. Hepatocellular carcinoma (HCC), the most prolific subtype, is the sixth most frequently diagnosed cancer and second leading cause of cancer-related mortality globally. ${ }^{23}$ Historically, $\mathrm{HCC}$ was most common in Asia and Africa, but in recent years the incidence in Western countries has been rising rapidly due to increased rates of hepatitis infection, alcohol abuse, obesity and diabetes, among other factors. $^{24}$

Early-stage HCC can be cured in 30\%-40\% of cases by surgical resection, transplantation, and ablative techniques. Unfortunately, nearly half of patients are diagnosed at an advanced stage when their tumor is unresectable and can't be cured by conventional SOC. The prognosis for these patients is especially poor - less than $10 \%$ OS at 5 years. ${ }^{25,26}$

Molecularly targeted therapies have been extensively investigated in HCC (Figure 3), with particular focus on the Ras-Raf-mitogen-activated protein kinase (MAPK) and phosphatidylinositol 3-kinase (PI3K)-Akt-mTOR pathways that play a central role in the development of HCC. In 2008, there was a significant advancement in the treatment of advanced stage disease with the approval of sorafenib, following demonstration of improved OS in phase 3 trials. ${ }^{27,28}$ Although sorafenib has become SOC for unresectable, nonablatable, advanced-stage HCC, it merely delays progression of disease and in most cases tumors begin to grow again after less than 6 months. This has created a need for therapies that improve on sorafenib efficacy or that can be used in the second-line setting after sorafenib failure.

A range of other molecularly targeted strategies have been used (Online, Table 4). The success of sorafenib, a multitargeted tyrosine kinase inhibitor (TKI) with anti-angiogenic properties, prompted intensive study of other TKIs with similar mechanism of action. As yet, though many 


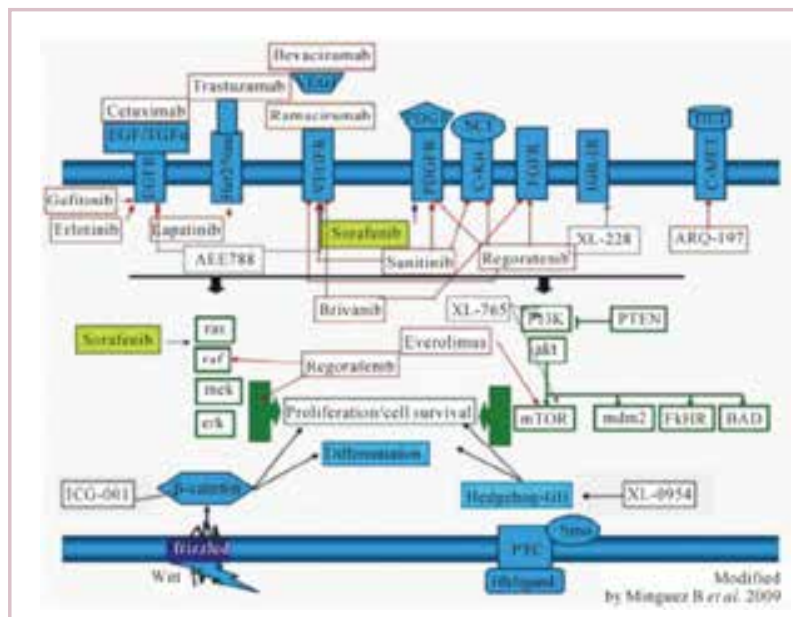

FIGURE 3 Molecularly targeted therapies tested in hepatocellular carcinoma. Reproduced with permission: Terry K, et al. Molecular targeted therapy of hepatocellular carcinoma. J Cancer Ther. 2013;4:426.

$B A D, B c l-2$-associated death receptor; EGF, epidermal growth factor; EGFR, EGF receptor; ERK, extracellular signal-related kinase; FGFR, fibroblast growth factor receptor; FkHR, forkhead transcription factor; HER2, human epidermal growth factor receptor 2; Hh, hedgehog; HGF, hepatocyte growth factor; IGF-1R, insulin-like growth factor receptor 1; MDM2, mouse double minute 2 homolog; MEK, mitogen-activated protein kinase kinase; mTOR, mammalian target of rapamycin; PDGF, platelet-derived growth factor; PDGFR, PDGF receptor; PI3K, phosphatidylinositol 3-kinase; PTC, patched; PTEN, phosphatase and tensin homolog; SCF, stem cell factor; Smo, smoothened; TGF $\alpha$ : transforming growth factor-alpha; VEGF, vascular endothelial growth factor; VEGFR, VEGF receptor

have reached phase 3 development, none have improved on the efficacy or tolerability of sorafenib in the first-line setting or shown benefit in the second-line setting.

Most recently, the results of the phase $3 \mathrm{REACH}$ trial of VEGFR2 inhibitor ramucirumab were presented at the annual meeting of the European Society of Molecular Oncology and showed no survival benefit. ${ }^{29}$ However, an abstract presented at the Gastrointestinal Cancers Symposium in early 2015 described a post hoc analysis of this study, suggesting that ramucirumab led to a greater reduction in the risk of death in patients with progressively higher baseline $\alpha$-fetoprotein (AFP) levels $(\geq 400 \mathrm{ng} / \mathrm{mL}$ ), as well as improved OS in all subgroups tested for patients with an AFP level $>400 \mathrm{ng} / \mathrm{mL}$. Studies are ongoing to understand this relationship. ${ }^{30}$

Many of the agents tested are more potent or selective than sorafenib and their failure to improve survival has led to the suggestion that sorafenib monotherapy has achieved a therapeutic ceiling and to move beyond this we need an improved understanding of the precise mechanism of action of these drugs or a focus on combination therapy. To this end, more than 45 clinical trials of sorafenib are currently recruiting, particularly in combination with other targeted agents. The results of a phase 3 study of sorafenib and the EGFR inhibitor erlotinib (SEARCH) were recently published, but the combination was not found to be significantly better than sorafenib monotherapy. ${ }^{31}$

Other targeted therapies with a different mechanism of action have also been evaluated, including inhibitors of mTOR, MET, IGF1R, MEK, and others. Some clinical trials of these agents are ongoing, but many have reported failures, including the recent phase 3 EVOLVE-1 trial of mTOR inhibitor everolimus in patients for whom sorafenib treatment had failed. ${ }^{32}$ Although the disease control rate was moderately improved, there was no OS benefit. A phase 3 trial of MET inhibitor tivantinib is ongoing (METIV-HCC) that is preselecting for patients with high levels of MET expression. In spite of early reports of a higher incidence of neutropenia in the MET inhibitor arm, a data monitoring committee recommended continuation of the trial in early 2014 with a lower dose of tivantinib. ${ }^{33}$ If successful, this would prove to be the first agent aimed at a biologically selected patient population in HCC.

\section{References}

1. Stupp R, Hegi ME, Mason WP, et al. Effects of radiotherapy with concomitant and adjuvant temozolomide versus radiotherapy alone on survival in glioblastoma in a randomized phase III study: 5-year analysis of the EORTC-NCIC trial. Lancet Oncol. 2009;10:459466.

2. Stupp R, Mason WP, van den Bent MJ, et al. Radiotherapy plus concomitant and adjuvant temozolomide for glioblastoma. $\mathrm{N}$ Engl J Med. 2005;352:987-996.

3. Cohen MH, Shen YL, Keegan P, et al. FDA drug approval summary: bevacizumab (Avastin) as treatment of recurrent glioblastoma multiforme. Oncologist. 2009;14:1131-1138.

4. Chinot OL, Wick W, Mason W, et al. Bevacizumab plus radiotherapy-temozolomide for newly diagnosed glioblastoma. New Engl J Med. 2014;370:709-722.

5. Gilbert MR, Dignam JJ, Armstrong TS, et al. A randomized trial of bevacizumab for newly diagnosed glioblastoma. New Engl J Med. 2014;370:699-708.

6. Batchelor TT, Mulholland P, Neyns B, et al. Phase III randomized trial comparing the efficacy of cediranib as monotherapy, and in combination with lomustine, versus lomustine alone in patients with recurrent glioblastoma. J Clin Oncol. 2013;31:3212-3218.

7. Stupp R, Hegi R, Gorlia T, et al. Cilengitide combined with standard treatment for patients with newly diagnosed glioblastoma with methylated MGMT promoter (CENTRIC EORTC 26071-22072 study): a multicentre, randomized, open-label, phase 3 trial. Lancet Oncol. 2014;15:1100-1108.

8. Rao SK, Edwards J, Joshi AD, et al. A survey of glioblastoma genomic amplifications and deletions. J Neurooncol. 2010;96:169-179.

9. Weller M, Kaulich K, Hentschel B, et al. Assessment and prognostic significance of the epidermal growth factor receptor vIII mutation in glioblastoma patients treated with concurrent and adjuvant temozolomide radiochemotherapy. Int J Cancer. 2014;134:2437-2447.

10. US Food and Drug Administration. Medical Devices: NovoTTF100A System. http://www.fda.gov/MedicalDevices/ProductsandMedicalProcedures/DeviceApprovalsandClearances/RecentlyApprovedDevices/ucm254480.htm. Updated September 6, 2013 Accessed March 1, 2015.

11. Stupp R, et al. Interim analysis of the EF-14 trial: a prospective, multicenter trial of NovoTTF-100A together with temozolomide compared to temozolomide alone in patients with newly diagnosed glio- 
blastoma multiforme. [SNO abstract]. Neuro Oncol. 2014;16(suppl 5):v167.

12. Xu LW, Chow KKH, Lim M, et al. Current vaccine trials in glioblastoma: a review [published online April 3, 2014]. J Immunol Res. doi. org $/ 10.1155 / 2014 / 796856$

13. Reardon D, Schuster J, Tran D, et al. ReACT: A Phase II study of rindopepimut vaccine $(\mathrm{CDX}-110)$ plus bevacizumab in relapsed glioblastoma. Neurooncol. 2014;16(suppl. 5):v116.

14. American Cancer Society. Cancer Facts and Figures 2015. http:// www.cancer.org/research/cancerfactsstatistics/cancerfactsfigures2015/ index. Accessed March 1, 2015.

15. Patel SR. Fifty years of advances in sarcoma treatment: moving the needle from conventional chemotherapy to targeted therapy. Am Soc Clin Oncol Educ Book. 2014:259-62.

16. Perry JA, Kiezun A, Tonzi P, et al. Complementary genomic approaches highlight the $\mathrm{PI} 3 \mathrm{~K} / \mathrm{mTOR}$ pathway as a common vulnerability in osteosarcoma. Proc Natl Acad Sci USA. 2014;111:E55645573.

17. Wagner MJ, Maki RG. Type 1 insulin-like growth factor receptor targeted therapies in pediatric cancer. Frontiers Oncol. 2013;3:article 9 .

18. Heymann D, Redini F. Targeted therapies for bone sarcomas. BoneKEY Rep. 2013;2:378

19. Forscher C, Mita M, Figlin R. Targeted therapy for sarcomas. Biologics Targets Ther. 2014;8:91-105.

20. Subbiah V and Kurzrock R. Ewing's sarcoma: overcoming the therapeutic plateau. Discov Med. 2012;13:405-415.

21. Chang DK, Grimmond SM, Evans TRJ, et al. Mining the genomes of exceptional responders. Nat Rev Cancer. 2014;14:291-2.

22. Subbiah V. Prospects and pitfalls of personalizing therapies for sarcomas: from children, adolescents, and young adults to the elderly. Curr Oncol Rep. 2014;16:401.

23. Stewart BW, Wild CP, ed. World Cancer Report 2014. Lyon, France: International Agency for Research on Cancer; 2014.
24. El-Serag HB. Epidemiology of hepatocellular carcinoma in USA. Hepatol Res. 2007;37(suppl 2):S88-S94.

25. Galuppo R, Ramaiah D, Ponte OM, et al. Molecular therapies in hepatocellular carcinoma: what can we target? Dig Dis Sci. 2014;59:1688-1697.

26. European Association for the Study of the Liver, European Organisation for Research and Treatment of Cancer. EASL-EORTC clinical practice guidelines: management of hepatocellular carcinoma. J Hepatol. 2012;56:908-943.

27. Llovet JM, Ricci S, Mazzaferro V, et al. Sorafenib in advanced hepatocellular carcinoma. N Engl J Med. 2008;359:378-390.

28. Cheng A-L, Kang Y-K, Chen Z, et al. Efficacy and safety of sorafenib in patients in the Asia-Pacific region with advanced hepatocellular carcinoma: a phase III randomised, double-blind, placebocontrolled trial. Lancet Oncol. 2009;10:25-34.

29. Zhu AX, et al. Ramucirumab as second-line treatment in patients with advanced HCC following first-line therapy with sorafenib [ESMO abstract LBA16]. Ann Oncol. 2014;25(suppl 5):v1-v41.

30. Zhu AX, Ryoo B-Y, Yen C-J, et al. Ramucirumab as second-line treatment in patients with advanced hepatocellular carcinoma: analysis of patients with elevated alpha-fetoprotein from the randomized phase III REACH study [2015 Gastrointestinal Cancers Symposium, abstract 232]. J Clin Oncol. 2015;33(suppl 3):abstr 232.

31. Zhu AX, Rosmorduc O, Evans TR, et al. SEARCH: A phase III, randomized, double-blind, placebo-controlled trial of sorafenib plus erlotinib in patients with advanced hepatocellular carcinoma. J Clin Oncol. 2015;33(6):559-566

32. Zhu AX, Kudo M, Assenat E, et al. Effect of everolimus on survival in advanced hepatocellular carcinoma after failure of sorafenib: the EVOLVE-1 randomized clinical trial. JAMA 2014;312:57-67.

33. ArQule. ArQule provides updates on clinical trials in hepatocellular carcinoma and non-small cell lung cancer with tivantinib. http:// investors.arqule.com/releasedetail.cfm?releaseid $=819847$. Released January 16, 2014. Accessed March 1, 2015. 